\title{
A Hidden Markov Model for Indoor Trajectory Tracking of Elderly People
}

\author{
Daniel Alshamaa, Aly Chkeir, Farah Mourad-Chehade \\ Charles Delaunay Institute \\ University of Technology of Troyes \\ Troyes, France \\ (daniel.alshamaa, aly.Chkeir, farah.chehade)@utt.fr
}

\author{
Paul Honeine \\ LITIS lab \\ University of Rouen Normandie \\ Rouen, France \\ paul.honeine@univ-rouen.fr
}

\begin{abstract}
Tracking of elderly people is indispensable to assist them as fast as possible. In this paper, we propose a new trajectory tracking technique to localize elderly people in real time in indoor environments. A mobility model is constructed, based on the hidden Markov models, to estimate the trajectory followed by each person. However, mobility models can not be used as standalone tracking techniques due to accumulation of error with time. For that reason, the proposed mobility model is combined with measurements from the network. Here, we use the power of the WiFi signals received from surrounding Access Points installed in the building. The combination between the mobility model and the measurements result in tracking of elderly people. Real experiments are realized to evaluate the performance of the proposed approach.
\end{abstract}

Index Terms-elderly people, hidden Markov models, mobility, tracking, WiFi RSSI.

\section{INTRODUCTION}

The number of elderly people is increasing worldwide. From 461 million people older than 65 years in 2004, it is expected that this number reaches 2 billion by 2050 [1]. By the same time, the United Nations expects that 64 countries will have an elderly population of more than $30 \%$ [2]. One of the major problems related to older population is an increased risk of adversse events, such as falls. According to the World Health Organization [3], more than $28 \%$ of people aged 65 and over fall each year, increasing to more than $32 \%$ for those over 70 years. When a fall happens, rapid intervention is needed in order to avoid serious injuries.

An indispensable condition for a fast intervention is the knowledge of the elderly person's location. This is applicable for any urgent assistance requested by elderly people in case of a fracture or moving inability in care centers, hospitals, nursing homes, etc. Elderly people might also have a difficulty in determining the location they are in, or how to arrive to the destination they desire [4]. The development of tracking techniques allows us to instantaneously determine the location of the elderly people at the moment they ask for help. This is also beneficial for the ambulance service and firefighters to assist people immediately in case of emergencies, through knowing their location instead of scanning the whole environment. The tracking algorithm can be executed in the background and displayed once the elderly person demands help or connected to the trigger of an alarm system. Real time localization is also required to study the Activities of Daily Living (ADL) in community-dwelling elderly people [5], [6].

A solution for localization is to equip each elderly person with a smart sensor in the form of a bracelet, a medallion, or smart phone. The objective becomes to localize the sensor, thus determining the position of the elderly person. Outdoor localization techniques are well-established using Global Positioning Systems (GPS) [7]. However, this technology can not be efficiently used for indoor applications [8]. Existing solutions consist in using signals that are available in closed environments, like ultra-wideband, Bluetooth, and WiFi [9], [10]. One advantage of WiFi signals over the others is that one can rely only on the Access Points (APs) present inside the building, with no additional hardware. The localization process consists then in finding the sensor's location according to the WiFi signals collected from APs [11], [12].

The tracking can be viewed as a sequential localization problem. Thus, it requires a real-time recursive location estimation algorithm. In addition to measurements collected from the network, one can use additional information such as knowledge of past location, speed, acceleration, or trajectory of the sensor being tracked, in order to correct the estimated location. Several state-of-the-art methods have been proposed to tackle this issue such as the Kalman filter [13], the extended Kalman filter (EKF) [14], and the particle filter [15].

In this paper, we propose a novel tracking approach for real time localization of vulnerable elderly people in indoor environments. The idea is to equip each person with a sensor that measures the Received Signal Strength Indicators (RSSIs) of WiFi APs. These measurements are used to build an observation model. A mobility model is then constructed to determine the trajectory of the sensor. It is based on hidden Markov models (HMMs), to be trained using information from the network. This mobility model is combined afterwards with the observation model in order to determine the location of the sensor, thus the elderly person.

The remainder of the paper is organized as follows. Section II states the problem. Section III describes the observation model. Section IV explains the mobility model based on the HMMs, and how it is combined with the observation model to track the sensors. Section V evaluates the performance of the proposed approach through experiments on real data. 


\section{Problem Statement}

The tracking problem consists in estimating the sensor's zone in real time using its mobility and the signals strengths it collects from the surrounding WiFi APs. We consider an environment composed of $N_{Z}$ zones, denoted by $Z_{j}, j \in$ $\left\{1, \ldots, N_{Z}\right\}$. Let $N_{A P}$ be the number of APs, denoted by $A P_{k}, \quad k \in\left\{1, \ldots, N_{A P}\right\}$, and $\rho_{t}$ be the vector of RSSI measurements collected by the sensor at the instant $t$ from surrounding APs. The aim of the proposed algorithm is to find a function $\mathbb{T}$, such that $\mathbb{T}\left(\boldsymbol{\rho}_{t}\right)=\left(\mathcal{W}_{t}\left(Z_{1}\right), \ldots, \mathcal{W}_{t}\left(Z_{N_{Z}}\right)\right)$, where $\mathcal{W}_{t}\left(Z_{j}\right)$ is the confidence level of having the sensor of observation $\rho_{t}$ residing in the zone $Z_{j}$ at the instant $t$. It is worth noting that only one sensor is considered here; however, the method can be applied in the same manner to as many sensors as needed.

\section{OBSERVATION MODEL}

The observation model uses RSSI data received from surrounding WiFi APs to estimate the zone of the mobile sensor. In an offline phase, fingerprints are collected by measuring the RSSIs of all existing APs in random positions of each zone. Let $\rho_{j, k, \ell}, \ell \in\left\{1, \ldots, N_{j}\right\}$, be the set of $N_{j}$ measurements collected in the zone $Z_{j}$ with respect to $A P_{k}$. Then, in the online phase, once a new RSSI measurement $\boldsymbol{\rho}_{t}$ of size $N_{A P}$ is received from all the detected APs, the model is used to assign certain evidence to each zone. Formally, the role of the observation model $\mathbb{O}$ is to assign a weight $m_{\mathbb{O}, t}(\cdot)$ to each zone $Z_{j}, j \in\left\{1, \ldots, N_{Z}\right\}$, for each observation $\boldsymbol{\rho}_{t}$ at instant $t$. To do that, the kernel density estimate is used to construct a distribution $Q(\cdot)$ by modeling the RSSIs of each zone with respect to all APs,

$$
Q_{Z_{j}}(\cdot)=\frac{1}{N_{j}} \sum_{\ell=1}^{N_{j}} \frac{1}{h_{j, 1} \ldots h_{j, N_{A P}}} \prod_{k=1}^{N_{A P}} \mathcal{K}\left(\frac{-\rho_{j, k, \ell}}{h_{j, k}}\right),
$$

where $\mathcal{K}(\cdot)$ is the kernel and $h_{j, k}$ is the bandwidth of the kernel in each zone with respect to each AP. Since the shape of the kernel does not effect the model [16], the Gaussian kernel is considered due to the facility of its analytical derivations,

$$
\mathcal{K}(u)=\frac{1}{\sqrt{2 \pi}} e^{-\frac{1}{2} u^{2}} .
$$

The bandwidth $h_{j, k}$ is estimated by maximizing the pseudolikelihood leave-one-out cross validation [17], $h_{j, k}=$ $\operatorname{argmax}_{h} M L_{j, k}(h)$, where $M L_{j, k}(h)$ is computed as,

$M L_{j, k}\left(h_{j, k}\right)=\frac{1}{N_{j}} \sum_{\ell=1}^{N_{j}} \log \left[\sum_{\ell^{\prime} \neq \ell} \mathcal{K}\left(\frac{\rho_{j, k, \ell^{\prime}}-\rho_{j, k, \ell}}{h_{j, k}}\right)\right]-\log \left[\left(N_{j}-1\right) h_{j, k}\right]$

It has been proven in literature that this criterion leads to a KDE that is the closest to the true model [18]. The weight assigned by the observation model to each zone at any instant $t$ is thus computed as the output of the kernel density estimate of each zone followed by a normalization phase,

$$
m_{\mathbb{O}, t}\left(Z_{j}\right)=\frac{Q_{Z_{j}}\left(\boldsymbol{\rho}_{t}\right)}{\sum_{i=1}^{N_{Z}} Q_{Z_{i}}\left(\boldsymbol{\rho}_{t}\right)}
$$

\section{HMM-BASED MOBILITY MODEL}

In this tracking approach, we construct a mobility model using hidden Markov models (HMMs). The idea is to create, for each transition between two consecutive zones, a trajectory that the sensor follows to move from one zone to another. A HMM is constructed for each trajectory, yielding a probability that the sensor has followed it. This probability is then combined with the evidence assigned by the observation model to determine a confidence level that the sensor resides in each zone. At first, we provide a general overview of the HMMs. We then present the architecture of the proposed mobility model, the way we use it to assign evidence, and the confidence-based zone estimation through combination with the observation model.

\section{A. Hidden Markov Models}

A HMM is a probabilistic model that can be used to represent a sequence of observations, and these observations can be either discrete or continuous, and can be either time dependent or independent [19]. The reason why the states in HMM are described as hidden is because the observer is unaware of the nature of the states. A first order HMM assumes that the current state depends only on the previous state. A second order HMM assumes that the present state depends on the two previous states, and a third order HMM considers three previous states and so on. Suppose we have an $N_{S}$-state HMM model $\Lambda$, where $N_{S}$ is the total number of states denoted $S=\left\{s_{1}, s_{2}, \ldots, s_{N_{S}}\right\}$. Whenever a sequence of length $\alpha, R=\left\{R_{1}, R_{2}, \ldots, R_{\alpha}\right\}$, is observed, the objective of the HMM is to determine the corresponding state sequence $S=\left\{s_{1}, s_{2}, \ldots, s_{\alpha}\right\}$.

An HMM is defined by three primary parameters, as described in the following. The first one is the transition probability that designates the probability of arriving at the next states for each present state. The set of probabilities of transitioning from any state to another one are termed as transition probabilities and form a matrix $\boldsymbol{A}$. Since the model is a HMM, the actual states are hidden from the observer. The latter measures observable data and then determines the probability that each type of observation is in each state. These probabilities are termed as emission probabilities and form a matrix $\boldsymbol{B}$. Another parameter of the HMM is $\pi$, which is the set of probabilities of starting at different states. It can be either uniform, random, or any vector generated from prior knowledge. Therefore, any HMM can be defined . as $\Lambda=(A, B, \pi)$. We denote $a_{f y}, f, y \in\left\{1, \ldots, N_{S}\right\}$, an element of the matrix $\boldsymbol{A}$, the transition probability from state $f$ to $y$. We denote $b_{f}(R), f=1, \ldots, N_{S}$, an element of the matrix $\boldsymbol{B}$, the output probability distribution [20].

\section{B. Architecture}

In this tracking approach, we make use of the trajectory of the sensor in indoor environments. The objective is to detect a transition of the sensor from any zone to another in a period of time. For that reason, we use the HMMs to determine a probability or likelihood that the sensor has 


\begin{tabular}{|c|c|c|}
\hline $\begin{array}{c}Z_{1} \\
\text { transitionzone } \\
\because \because \because \because:\end{array}$ & $Z_{2}$ & $Z_{3}$ \\
\hline$\frac{Z_{4}}{\because \because::}$ & $\begin{array}{c}Z_{5} \\
: \because: \because: \text { : }\end{array}$ & $Z_{6}$ \\
\hline$Z_{7}$ & $Z_{8}$ & $Z_{9}$ \\
\hline
\end{tabular}

(a) Construction of transition region between all pair of neighboring zones. transition zone

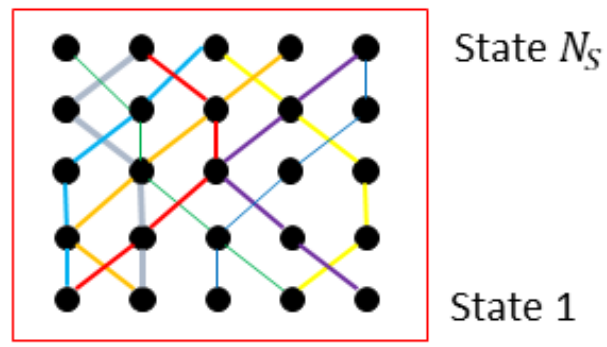

(b) Creation of $N_{S}$-state HMM from trajectories in each transition region.

Fig. 1: Illustration of the architecture of the HMM-based mobility model.

followed a certain trajectory. Each HMM $\Lambda$ is defined by the three aforementioned parameters, $\Lambda=(A, B, \pi)$. As a state sequence $S=\left\{s_{1}, \ldots, s_{\alpha}\right\}$ is determined, we can observe a sequence $R=\left\{R_{1}, \ldots, R_{\alpha}\right\}$ since the states are hidden, corresponding to a vector of RSSI measurements at each state. We are interested in determining the probability $P(R \mid \Lambda)$, which is the probability of observing the sequence $R$, given the HMM model $\Lambda$. This probability is used as an evidence to be combined with that obtained by the observation model $\mathbb{O}(\cdot)$ to determine a confidence level of having the sensor residing in each zone.

We construct a set of HMMs denoted as $\Lambda_{i j}, i, j \in$ $\left\{1, \ldots, N_{Z}\right\}$, where $\Lambda_{i j}$ is an $N_{S}$-state HMM corresponding to a transition region or trajectory between zones $Z_{i}$ and $Z_{j}$. The parameter $N_{S}$ is the number of states chosen by the user in each transition region. In the offline phase, a transition region between each pair of neighboring zones is created as shown in Fig. 1(a). This region is divided into $N_{S}$ states. At each state, a set of $\eta$ RSSI measurements is collected. This leads to a set of $\kappa=N_{S} \times \eta$ RSSI measurements in each transition region. Trajectories or sequences are constructed by randomly selecting a measurement from each state, as shown in Fig. 1(b). All these constructed trajectories are considered as a database for each HMM. The parameters of each HMM $\Lambda_{i j}=(A, B, \pi)$ are calculated as follows,

- Since at each state, except for the first and the last where there is only two options, the sensor can equiprobably stay in its position, move to the state upfront, or move to the state behind, we define the $N_{S} \times N_{S}$ transition matrix $A$ as,

$$
A=\left(\begin{array}{cccccc}
\frac{1}{2} & \frac{1}{2} & 0 & 0 & \ldots & 0 \\
\frac{1}{3} & \frac{1}{3} & \frac{1}{3} & 0 & \ldots & 0 \\
0 & \frac{1}{3} & \frac{1}{3} & \frac{1}{3} & \ldots & 0 \\
\vdots & \vdots & \vdots & \vdots & \ddots & \vdots \\
0 & 0 & 0 & \ldots & \frac{1}{2} & \frac{1}{2}
\end{array}\right)
$$

- The emission model of each sequence is computed by modeling the offline collected RSSI measurements of each sequence with a multi-dimensional distribution, $b_{f}(R)=Q_{\kappa}\left(\rho_{f}\right), i \in\left\{1, \ldots, N_{Z}\right\}$. The distribution
$Q_{\kappa}\left(\rho_{f}\right)$ is the output of the distribution $Q(\cdot)$ for observation $\rho_{f}$ modeled using the RSSIs $\kappa$ with respect to all the detected APs.

- Unless there is a prior knowledge regarding the starting state of the sensor, the vector $\pi$ is defined as $\pi=$ $\left[\frac{1}{N_{S}}, \ldots, \frac{1}{N_{S}}\right]$.

\section{Weight assignment}

Given a $N_{S}$-state HMM model $\Lambda$, and an observation sequence $R$, the aim is to evaluate the probability of observing the sequence $P(R \mid \Lambda)$. This is a problem of evaluating the observed sequence when we know the parameters of the HMM. In the simplest form, we can break down the evaluation of $P(R \mid \Lambda)$ as follows. Given a state sequence $S=\left\{s_{1}, \ldots, s_{\alpha}\right\}, 1 \leq \alpha \leq N_{S}$, we can compute the joint probability of the observed sequence and the state sequence,

$$
P(R, S \mid \Lambda)=P(R \mid S, \Lambda) \times P(S \mid \Lambda) .
$$

This is the product of the probability of the observation sequence $R$ given $S$, and the probability of the state sequence $S$ given the model. The first term is obtained from the emission matrix $B$ as,

$$
P(R \mid S, \Lambda)=\prod_{f=1}^{\alpha} b_{s_{f}}\left(R_{f}\right) .
$$

The second term is obtained from the transition matrix $A$ as,

$$
P(S \mid \Lambda)=\prod_{f=1}^{\alpha} a_{s_{f-1} s_{f}} .
$$

We can then derive $P(R \mid \Lambda)$ as the summation of $P(R, S \mid \Lambda)$ over all possible state sequences $S$ [21],

$$
P(R \mid \Lambda)=\sum_{\text {for all } S} P(R, S \mid \Lambda)=\sum_{\text {for all } S} \prod_{f=1}^{\alpha} a_{s_{f-1}} a_{s_{f}} b_{s_{f}}\left(R_{f}\right) .
$$

The total number of state paths increases quickly with the length of the sequence, and thus becomes computationally expensive and not feasible depending on $\alpha$. However, a forward-backward algorithm can be used to obtain $P(R \mid \Lambda)$, and 
reduces the computational burden. The expression in equation (8) can be transformed as follows,

$P(R \mid \Lambda)=\sum_{y=1}^{N_{S}} P\left(R_{1}, \ldots, R_{f}, s_{f}=y \mid \Lambda\right) \cdot P\left(R_{f+1}, \ldots, R_{\alpha} \mid s_{f}=y, \Lambda\right)$.

The probability of observing the sequence can thus be determined using forward and backward probabilities,

$$
\begin{gathered}
g_{f}(y)=P\left(R_{1}, \ldots, R_{f}, s_{f}=y \mid \Lambda\right) . \\
h_{f}(y)=P\left(R_{f+1}, \ldots, R_{\alpha} \mid s_{f}=y, \Lambda\right) .
\end{gathered}
$$

The probabilities in equations (10) and (11) can be computed recursively,

$$
\begin{gathered}
g_{f+1}(y)=\left[\sum_{x=1}^{N_{S}} g_{f}(x) a_{x y}\right] b_{y}\left(R_{f+1}\right) \\
h_{f}(y)=\sum_{x=1}^{N_{S}} a_{y x} b_{x}\left(R_{f+1}\right) h_{f+1}(x) .
\end{gathered}
$$

Therefore, the probability $P(R \mid \Lambda)$ is given by,

$$
P(R \mid \Lambda)=\sum_{y=1}^{N_{S}} g_{f}(y) h_{f}(y) .
$$

This is the probability of observing a sequence $R$ of length $\alpha$ given an $N_{S}$-states HMM $\Lambda$.

The objective of the proposed mobility model is to assign an evidence that the sensor has followed a trajectory, which is a transition between a zone and another. After constructing a HMM $\Lambda_{i j}$ for each transition between two zones as explained in the previous paragraph, it is time to use these HMMs to estimate the trajectory of the sensors. Once a sequence $R=\left\{R_{1}, \ldots, R_{\alpha}\right\}$ is detected, each HMM $\Lambda_{i j}$ assigns a probability or likelihood that the sensor has followed the state sequence corresponding to that HMM. The probabilities $P\left(R \mid \Lambda_{i j}\right), i, j \in\left\{1, \ldots, N_{Z}\right\}$, are computed for each HMM. Thus, the probability of transitioning from any zone to another is computed. For pairs of zones where no transition is possible, the probability is zero. We define the transition coefficient $p_{i j}, i, j \in\left\{1, \ldots, N_{Z}\right\}$, from $Z_{i}$ to $Z_{j}$ as follows,

$$
p_{i j}= \begin{cases}P\left(R \mid \Lambda_{i j}\right), & \text { if } i \neq j ; \\ 1-\sum_{j=1}^{N_{Z}} P\left(R \mid \Lambda_{i j}\right), & \text { if } i=j .\end{cases}
$$

We compute $p_{i j}$ for $i=j$ as such, because we consider that the probability of the sensor staying in the same zone to be the complement of all the probabilities that the sensor moves from that zone to all other zones. We compute the evidence $m_{\mathbb{M}, t}(\cdot)$ as follows,

$$
m_{\mathbb{M}, t}\left(Z_{j}\right)=\sum_{i=1}^{N_{Z}} m_{\mathbb{O}, t-1}\left(Z_{i}\right) \times p_{i j},
$$

where $m_{\mathbb{O}, t-1}\left(Z_{i}\right)$ is the weight associated by the observation model $\mathbb{O}(\cdot)$ at instant $t-1$. If there is no transition from a zone to another, the coefficient $p_{i j}$ for $i=j$ will be large.
This does not cause a problem when used in equation (16) because, if there is no transition, all coefficients $p_{i j}$ for $i=j$ will be large, and thus $m_{\mathbb{O}, t-1}(\cdot)$ is the deciding evidence. The zone already having a high confidence in the previous instant will still have high confidence, relative to the other zones. The determination of $p_{i j}$ for $i=j$, as done here is only valid by the way we transfer this probability to the next instant, and its combination with the observation model. In fact, if there is no transition, we will obtain large transition coefficients $p_{i i}$, for all $i \in\left\{1, \ldots, N_{Z}\right\}$, which results in false estimations.

\section{Confidence-based zone estimation}

The confidence level $\mathcal{W}_{t}(\cdot)$ that the sensor resides in each zone is obtained by combining the evidence assigned by both the observation and the mobility models as follows,

$$
\mathcal{W}_{t}\left(Z_{j}\right)=\frac{m_{\mathbb{Q}, t}\left(Z_{j}\right) \times m_{\mathbb{M}, t}\left(Z_{j}\right)}{\sum_{\chi=1}^{N_{Z}} m_{\mathbb{O}, t}\left(Z_{\chi}\right) \times m_{\mathbb{M}, t}\left(Z_{\chi}\right)} .
$$

This zone having the highest confidence level is then selected. A second choice is also possible by choosing the zone having th second highest evidence.

\section{EXPERIMENTAL RESUlTS}

In this section, we evaluate the performance of the proposed tracking approach through experiments in a real environment. We study the influence of the mobility model when combined with the observation model. We then study the influence of the sequence length $\alpha$. In addition, we compare the performance of the proposed approach with state-of-the-art techniques.

\section{A. Experimental Setup}

Experiments are conducted in WLAN environments, in the Statistics and Operational Research Department and the Living Lab of the University of Technology of Troyes, France. The layout plan of Experiment 1 has an approximated area of $500 \mathrm{~m}^{2}$, and is partitioned into 21 zones. The layout plan of Experiment 2 has an area of $550 \mathrm{~m}^{2}$ and is partitioned to 19 zones. The experiments are not realized on elderly people, but on healthy adults taking into consideration the situation and movement of elderly people. To evaluate the performance of the mobility-based tracking approach, 10 trajectories of 50 observations each are examined. In order to consider an environment similar to that of elderly people, their movement is studied. Bohannon [22] studied the gait speed of 230 healthy adults aged between 20 and 79 years, measured attheir usual pace. The study revealed the following results. The mean comfortable gait speed for women in their 70s was found to be $1.27 \mathrm{~m} / \mathrm{s}$, while it wa $1.33 \mathrm{~m} / \mathrm{s}$ for men. Montero-Odasso et al. [23] explored the gait speed of 100 elderly patients aged 75 years and over. A maximum gait speed was found to be $0.8 \mathrm{~m} / \mathrm{s}$. Graham et al. [24] examined the walking speed of 174 in-hospital elderly people aged 65 years and over. The study reported an average velocity of $0.43 \mathrm{~m} / \mathrm{s}$ of hospitalized older adults. As demonstrated by various studies, the walking speed varies with sex, height, age, health, country, etc [22], 
TABLE I: Influence of the mobility model on the performance of the tracking approach.

\begin{tabular}{lccccc}
\hline & \multicolumn{2}{c}{ Experiment 1 } & & \multicolumn{2}{c}{ Experiment 2 } \\
\cline { 2 - 3 } \cline { 5 - 6 } Model & accuracy $(\%)$ online time $(\mathrm{s})$ & accuracy $(\%)$ & online time (s) \\
\hline Observation model & 79.77 & 0.1104 & & 80.44 & 0.1479 \\
Tracking approach & $\mathbf{8 7 . 7 4}$ & 0.1944 & & $\mathbf{8 9 . 4 3}$ & 0.2138 \\
\hline
\end{tabular}

[25]. We consider here an average speed of $1 \mathrm{~m} / \mathrm{s}$, then we study the influence of this parameter on the performance of the proposed approach. In fact, this speed is important to construct the $N_{S}$ state HMMs. In a $1 \mathrm{~m} / \mathrm{s}$ setting and $0.75 \mathrm{~s}$ as the time interval between two localization instants, we can construct $N_{S}$ states where the distance between each two states is $d_{S}=1 \mathrm{~m} / \mathrm{s} \times 0.75 \mathrm{~s}=0.75 \mathrm{~m}$. Although this method can be used to track any sensor in general or person in particular, it is more convenient and efficient for tracking elderly people. In fact, the relatively low speed of these people allows us to build longer HMMs, thus collecting more information about the trajectory of the sensors.

\section{B. Evaluation of performance}

We study the influence of the mobility model on the performance of the tracking approach when combined with the observation model, for an average speed of $1 \mathrm{~m} / \mathrm{s}$. In each transition region between neighboring zones, a 10-state HMM is created. The chosen length of the observed sequence $\alpha$ varies then from 1 to $N_{S}=10$. We consider here a sequence length $\alpha=8$, as for a sequence length $\alpha=10$, the gained accuracy is not worthy the added processing time. This means that after receiving a sequence of 8 observations, we use the HMMs to determine the probability that the sensor has followed the 8-state sequence of the 10-state HMMs. Table I shows the influence of the mobility model on the performance of the tracking approach when combined with the observation model. As the table shows, a significant enhancement in the overall accuracy is noted when the observation model is combined with the proposed mobility model, yet at the expense of an additional processing time.

We study the influence of the length of the sequence $\alpha$ by varying it from 2 to 10 and evaluating the performance of the tracking approach. Table II shows the influence of the sequence length $\alpha$ on the overall accuracy and the execution time. As the table shows, the overall accuracy of the tracking algorithm increases as the sequence length increases. This is due to augmenting the amount of information used by the HMMs to determine the likelihood that the sensor has followed the different trajectories. However, enlarging the sequence length increases the execution time. After a sequence of length 8 , no significant accuracy is gained, yet with an important additional execution time. For that reason, we consider only sequences of length 8 .
TABLE II: Influence of the sequence length $\alpha$ on the performance of the tracking approach.

\begin{tabular}{lccccc}
\hline & \multicolumn{2}{c}{ Experiment 1 } & & \multicolumn{2}{c}{ Experiment 2 } \\
\cline { 2 - 3 } \cline { 5 - 6 }$\alpha$ & accuracy (\%) & online time (s) & & accuracy (\%) & online time (s) \\
\hline 2 & 80.60 & 0.1308 & & 81.81 & 0.1507 \\
4 & 82.19 & 0.1430 & & 84.22 & 0.1662 \\
6 & 84.42 & 0.1725 & & 87.76 & 0.1877 \\
8 & 87.74 & 0.1944 & & 89.43 & 0.2138 \\
10 & $\mathbf{8 7 . 9 7}$ & 0.2486 & & $\mathbf{9 0 . 5 4}$ & 0.2503 \\
\hline
\end{tabular}

\section{Comparison with state-of-the-art methods}

In this paragraph, the proposed approach is compared with well-known localization techniques. Koyuncu \& Yang [26] present a weighted $k$-nearest neighbors algorithm (WKNN) for indoor localization. To estimate the position of the sensor, the new received measurement is compared with the elements in the fingerprint database using Euclidean distances. A set of $k$ smallest Euclidean distances is selected and the $k$-nearest neighbors algorithm is then applied. The algorithm averages the coordinates of the $k$-nearest neighbors of the sensor, weighting each distance by a factor previously determined according to a mathematical model, to give its location estimate. Shang et al. [27] propose a connectivity-based localization algorithm. The advantage of connectivity-based algorithms is that they do not rely on collected measurements. The sensor's location is given as the intersection of the ranges of the APs detected by the sensor. On the other hand, conventional classification techniques such as neural network (NN) [28] and Support Vector Machines (SVM) [29] are applied. Table III compares the overall accuracy and the localization processing time of the various described techniques. As the table shows, the proposed approach outperforms other state-of-the-art methods in terms of overall accuracy. This is at the expense of a higher processing time. Another advantage of the proposed method is that it does not require an inertial measurement unit to measure the speed. This makes it advantageous with respect to the existing statistical filters presented in the introduction, where the instantaneous inertial measurements are required to update the estimation.

TABLE III: Comparison of the proposed approach with various localization methods.

\begin{tabular}{lccccc}
\hline & \multicolumn{2}{c}{ Experiment 1} & & \multicolumn{2}{c}{ Experiment 2 } \\
\cline { 2 - 3 } \cline { 5 - 6 } Method & accuracy $(\%)$ & online time $(\mathrm{s})$ & accuracy $(\%)$ & online time $(\mathrm{s})$ \\
\hline WKNN & 83.78 & 0.0982 & & 84.28 & 0.1265 \\
Connectivity & 84.29 & 0.1107 & & 86.67 & 0.1338 \\
NN & 84.72 & 0.1466 & & 85.82 & 0.1866 \\
SVM & 85.55 & 0.1559 & & 86.47 & 0.1912 \\
Proposed & $\mathbf{8 7 . 7 4}$ & 0.1944 & & $\mathbf{9 0 . 5 4}$ & 0.2503 \\
\hline
\end{tabular}




\section{CONCLUSION AND PERSPECTIVES}

In this paper, we presented a mobility-based tracking approach for elderly people in indoor environments. The mobility model is based on hidden Markov models and aims at determining the trajectory that the elderly person follows. The proposed mobility model was combined with an observation model to determine the location of the elderly person. The performance of the tracking approach was illustrated through experiments in two real scenarios. The conducted experiments demonstrated significant enhancement in the overall accuracy carried by the mobility model. The accuracy of the tracking approach was also found to increase with the sequence length. In addition to the enhancement in the overall accuracy, an important advantage of the proposed approach, as compared with the state-of-the-art tracking techniques, is that no inertial measurement units are needed to perform the tracking. In future work, we aim at combining the proposed mobility model with other more advanced observation model to achieve higher tracking accuracy. In addition, we will conduct the experiments on elderly people instead of healthy adults with conditions similar to elderly people. This leads to a more appropriate evaluation of the proposed tracking approach. Moreover, we will use the described real-time tracking method to evaluate the ADL of elderly people.

\section{ACKNOWLEDGEMENT}

The authors would like to thank the European Regional Development Fund and the Grand Est region in France for funding this work. The authors also thank the Statistics and Operational Research Department and the Living Lab at the University of Technology of Troyes in France, for allowing them to realize the experiments.

\section{REFERENCES}

[1] A. Clegg, J. Young, S. Iliffe, M. O. Rikkert, and K. Rockwood, "Frailty in elderly people," The lancet, vol. 381, no. 9868, pp. 752-762, 2013.

[2] J. Guzman, A. Pawliczko, S. Beales, C. Till, and I. Voelcker, "Ageing in the twenty-first century: A celebration and a challenge," New York: United Nations Population Fund, 2012.

[3] W. H. Organization, Global tuberculosis control: surveillance, planning, financing: WHO report 2008, vol. 393. World Health Organization, 2008.

[4] C. Tsirmpas, A. Rompas, O. Fokou, and D. Koutsouris, "An indoor navigation system for visually impaired and elderly people based on radio frequency identification (RFID)," Information Sciences, vol. 320, pp. 288-305, 2015.

[5] J. Vermeulen, J. C. Neyens, E. van Rossum, M. D. Spreeuwenberg, and L. P. de Witte, "Predicting ADL disability in community-dwelling elderly people using physical frailty indicators: a systematic review," BMC geriatrics, vol. 11, no. 1, p. 33, 2011.

[6] M. Stikic, T. Huynh, K. Van Laerhoven, and B. Schiele, "ADL recognition based on the combination of RFID and accelerometer sensing," in International Conference on Pervasive Computing Technologies for Healthcare, pp. 258-263, IEEE, 2008.

[7] M. A. Al-Khedher, "Hybrid GPS-GSM localization of automobile tracking system," International Journal of Computer Science \& Information Technology, vol. 3, no. 6, pp. 75-85, 2011.

[8] T. Oshin, S. Poslad, and A. Ma, "Improving the energy-efficiency of GPS based location sensing smartphone applications," in IEEE International Conference on Trust, Security and Privacy in Computing and Communications (TrustCom), pp. 1698-1705, June 2012.
[9] H. Liu, H. Darabi, P. Banerjee, and J. Liu, "Survey of wireless indoor positioning techniques and systems," IEEE Transactions on Systems, Man, and Cybernetics, Part C: Applications and Reviews, vol. 37, pp. 1067-1080, Nov 2007.

[10] A. Disha, "A comparative analysis on indoor positioning techniques and systems," International Journal of Engineering Research and Applications, vol. 3, pp. 1790-1796, 2013.

[11] D. Alshamaa, F. Mourad-Chehade, and P. Honeine, "Zoning-based localization in indoor sensor networks using belief functions theory," in IEEE International Workshop on Signal Processing Advances in Wireless Communications (SPAWC), pp. 1-5, IEEE, 2016.

[12] D. Alshamaa, F. Mourad-Chehade, and P. Honeine, "Localization of sensors in indoor wireless networks: An observation model using WiFi RSS," in IFIP International Conference on New Technologies, Mobility and Security (NTMS), pp. 1-5, 2018.

[13] S. Mahfouz, F. Mourad-Chehade, P. Honeine, J. Farah, and H. Snoussi, "Target tracking using machine learning and Kalman filter in wireless sensor networks," IEEE Sensors Journal, vol. 14, no. 10, pp. 3715-3725, 2014.

[14] L. Zhang, Y. H. Chew, and W.-C. Wong, "A novel angle-of-arrival assisted extended kalman filter tracking algorithm with space-time correlation based motion parameters estimation," in International Wireless Communications and Mobile Computing Conference (IWCMC), pp. 1283-1289, IEEE, 2013.

[15] F. Hong, Y. Zhang, Z. Zhang, M. Wei, Y. Feng, and Z. Guo, "WaP: Indoor localization and tracking using WiFi-assisted particle filter," in IEEE Conference on Local Computer Networks (LCN), pp. 210-217, IEEE, 2014.

[16] A. Eckert-Gallup and N. Martin, "Kernel density estimation (KDE) with adaptive bandwidth selection for environmental contours of extreme sea states," in OCEANS MTS/IEEE Monterey, pp. 1-5, IEEE, 2016.

[17] J. Habbema and J. Hermans, "Selection of variables in discriminant analysis by F-statistic and error rate," Technometrics, vol. 19, no. 4, pp. 487-493, 1977.

[18] P. Hall, "Cross-validation in density estimation," Biometrika, vol. 69, no. 2 , pp. $383-390,1982$.

[19] S. R. Eddy, "Hidden markov models," Current Opinion in Structural Biology, vol. 6, no. 3, pp. 361-365, 1996.

[20] M. Oudelha and R. N. Ainon, "HMM parameters estimation using hybrid baum-welch genetic algorithm," in International Symposium in Information Technology (ITSIM), pp. 542-545, IEEE, 2010.

[21] Y. Yen, M. Fanty, and R. Cole, "Speech recognition using neural networks with forward-backward probability generated targets," in IEEE International Conference on Acoustics, Speech, and Signal Processing (ICASSP), vol. 4, pp. 3241-3244, IEEE, 1997.

[22] R. W. Bohannon, "Comfortable and maximum walking speed of adults aged 20-79 years: reference values and determinants," Age and Ageing, vol. 26, no. 1, pp. 15-19, 1997.

[23] M. Montero-Odasso, M. Schapira, C. Varela, C. Pitteri, E. R. Soriano, R. Kaplan, L. A. Camera, and L. Mayorga, "Gait velocity in senior people an easy test for detecting mobility impairment in community elderly," Journal of Nutrition Health and Aging, vol. 8, no. 5, pp. 340343, 2004.

[24] J. E. Graham, S. R. Fisher, I.-M. Bergés, Y.-F. Kuo, and G. V. Ostir, "Walking speed threshold for classifying walking independence in hospitalized older adults," Physical Therapy, vol. 90, no. 11, pp. 15911597, 2010.

[25] B. D. Capistrant, M. M. Glymour, and L. F. Berkman, "Assessing mobility difficulties for cross-national comparisons: results from the world health organization study on global ageing and adult health," Journal of the American Geriatrics Society, vol. 62, no. 2, pp. 329$335,2014$.

[26] H. Koyuncu and S. H. Yang, "A 2D positioning system using WSNs in indoor environment," International Journal of Electrical and Computer Sciences, vol. 11, no. 3, pp. 70-77, 2011.

[27] Y. Shang, W. Rumi, Y. Zhang, and M. Fromherz, "Localization from connectivity in sensor networks," IEEE Transactions on Parallel and Distributed Systems, vol. 15, no. 11, pp. 961-974, 2004.

[28] H. Dai, W.-h. Ying, and J. Xu, "Multi-layer neural network for received signal strength-based indoor localisation," IET Communications, vol. 10 , no. 6, pp. 717-723, 2016.

[29] L. Zhang, Y. Li, Y. Gu, and W. Yang, "An efficient machine learning approach for indoor localization," China Communications, vol. 14, no. 11, pp. 141-150, 2017. 